\title{
Multisensor data and thermodynamic sea-ice model based sea-ice thickness chart with application to the Kara Sea, Arctic Russia
}

\author{
M. SIMILÄ, M. MÄKYNEN, B. CHENG, E. RINNE \\ Finnish Meteorological Institute, Marine Research Unit, Ice Research Group, Helsinki, Finland \\ E-mail: markku.simila@fmi.fi
}

\begin{abstract}
We introduce a method to construct an ice thickness chart using two satellite datasets and a one-dimensional high-resolution thermodynamic snow and sea-ice model (HIGHTSI). Thin-ice thickness up to $40 \mathrm{~cm}$ is retrieved using MODIS ice surface temperature data. For thicker ice, thickness is retrieved from a combination of SAR image and background level ice thickness chart $\boldsymbol{H}_{\mathrm{i}}$ provided by HIGHTSI. Because $H_{\mathrm{i}}$ is inherently static, we utilize passive microwave data based sea-ice concentration charts to introduce mesoscale ice dynamics into $H_{\mathrm{i}}$. In order to create the thickness chart, the value of modified $H_{\mathrm{i}}$ is scaled by a factor which depends on the magnitude of backscattering coefficient $\sigma^{0}$. The proposed method is effective in cold winter conditions. We calculated a series of ice thickness charts covering the Kara Sea, Arctic Russia, during winter 2008/09. The ice thickness charts were validated against Russian ice charts. Our method gave realistic results in the thickness range $30-120 \mathrm{~cm}$ and was uncertain for the detection of thinner-ice areas. Due to the limitations in our reference dataset, more validation work is needed to establish the accuracy in more detail. Our thickness charts can be used for operational purposes and in climate studies.
\end{abstract}

\section{INTRODUCTION}

Reliable, up-to-date information on rapidly changing sea-ice conditions is essential for winter shipping and offshore operations in the Kara Sea, Arctic Russia. The most important sea-ice parameters are the location of the sea-ice edge, seaice thickness and degree of ice deformation. For the Kara Sea, information on sea-ice thickness is currently only available in mesoscale $(\sim 30-50 \mathrm{~km})$ spatial resolution, mainly in the form of manual ice charts. In this paper, we propose a new method to create a sea-ice thickness chart using three satellite sensors and a thermodynamic snow/sea-ice model. In the chart the ice cover is divided into several ice thickness categories for level ice and deformed ice fields. The constructed ice charts can also be applied in monitoring the ice season evolution in the Kara Sea in the context of climate studies. The work continues and extends that reported in Mäkynen and others (2010a,b). Mäkynen and others (2013) and Cheng and others (2013) are companion papers to this one.

The basic idea in the method proposed here is to retrieve the thin $(0-40 \mathrm{~cm})$ and thick $(>40 \mathrm{~cm})$ ice thickness categories separately using different satellite sensors. The thin-ice thickness is retrieved from the Moderate Resolution Imaging Spectroradiometer (MODIS), ice surface temperature data and numerical weather prediction (NWP) model HIgh-Resolution Limited Area Model (HIRLAM) data (Källén, 1996; Unden and others, 2002). The MODIS-based thickness retrieval has been assessed to be accurate up to $40 \mathrm{~cm}$ in typical winter weather conditions in our test area (Mäkynen and others, 2013). Thus we use $40 \mathrm{~cm}$ as our threshold value for the MODIS-based thickness. When the MODIS-based thickness chart, $h_{\mathrm{i}}^{\mathrm{M}}$, indicates that ice is thicker than $40 \mathrm{~cm}$, the sea-ice thickness, $h_{\mathrm{i}}$, is retrieved from a combination of Envisat Advanced Synthetic Aperture Radar (ASAR) Wide Swath Mode (WSM) imagery and background level ice thickness chart, $H_{\mathrm{b}}$, obtained through modification from $H_{\mathrm{i}}$ ice thickness field provided by the onedimensional HIGH-resolution Thermodynamic snow and Sea Ice (HIGHTSI) process model (Launiainen and Cheng,
1998; Cheng and others, 2003, 2008). The HIGHTSI model is forced by HIRLAM data. The applied modification to $H_{\mathrm{i}}$ utilizes sea-ice concentrations retrieved from Advanced Microwave Scanning Radiometer-Earth Observing System (AMSR-E) radiometer data. Using a novel approach we extract information on mesoscale ice dynamics from daily AMSR-E sea-ice concentration charts. Our method is demonstrated by producing a set of ice thickness charts for the Kara Sea in winter 2008/09.

When one examines the C-band backscattering coefficient $\left(\sigma^{0}\right)$ statistics as a function of ice thickness in the cold conditions of the Kara Sea, the distribution of $\sigma^{0}$ values for thin ice $(<30 \mathrm{~cm})$ covers most of the dynamic range of $\sigma^{0}$ (Fig. 1). However, we are now able to exclude thin-ice regions with the MODIS charts $h_{i}^{\mathrm{M}}$. This significantly reduces the ambivalence in the interpretation of $\sigma^{0}$. In closed drift-ice fields, $\sigma^{0}$ correlates on average with the ice thickness up to a saturation point.

We have validated our thickness chart using almost simultaneous Russian Arctic and Antarctic Research Institute (AARI) ice charts (Bushuev and Loshchilov, 2007) as our reference data. Our primary objective is to locate the areas of thinnest ice among the drift ice. The resulting ice charts do not attempt to estimate the total ice mass but the spatial distribution of thin and thick ice.

In the next section we briefly discuss earlier spaceborne remote-sensing approaches to retrieval of sea-ice thickness $h_{\mathrm{i}}$. We present the datasets at our disposal in Section 3. The proposed ice thickness retrieval method is introduced in Section 4. This is followed by some demonstration cases to illustrate the method. We then estimate the accuracy of our ice charts. Finally, we discuss the applicability of the method.

\section{EARLIER STUDIES OF SATELLITE-BASED SEA-ICE THICKNESS MAPPING}

Sea-ice thickness can be retrieved based on Archimedes' principle and satellite altimeter measurements of freeboard 




Fig. 1. Probability density functions (PDFs) of the backscattering coefficients $\sigma^{0}$ from Envisat ASAR WSM images for four different ice types. The $\sigma^{0}$ value is the average over a $3.1 \times 3.1 \mathrm{~km}^{2}$ area. Nilas (thickness $<10 \mathrm{~cm}$ ) and young ice $(\mathrm{Yl} ; 10-30 \mathrm{~cm}$ ) were identified on the basis of MODIS-based ice thickness charts. FYI is first-year ice.

(Laxon and others, 2003; Kwok and Rothrock, 2009). However, this method results in large relative errors for ice thinner than $1 \mathrm{~m}$ (Laxon and others, 2003). Passive microwave radiometer data at frequencies of 37 and 85 or $89 \mathrm{GHz}$ have been used to estimate thickness of thin ice up to 10$20 \mathrm{~cm}$ based on correlation between ice surface salinity (i.e. dielectric properties) and $h_{\mathrm{i}}$ (Martin and others, 2004; Nihashi and others, 2009; Tamura and Ohshima, 2011). Recently, Kaleschke and others (2012) demonstrated that lower-frequency L-band radiometer data from the Soil Moisture and Ocean Salinity (SMOS) satellite can be used to retrieve $h_{\mathrm{i}}$ up to $0.5 \mathrm{~m}$. The major drawbacks are the coarse resolution of the radiometer data, grid size from 12.5 to $35 \mathrm{~km}$, which prevents detection of leads and smaller polynyas, and the currently poorly known effect of snow cover on the thin-ice thickness estimation. Estimation of thin-ice thickness can also be conducted using satellite thermal imagery (e.g. MODIS)-based ice surface temperature, $T_{\mathrm{s}}$, together with atmospheric forcing data through the ice surface heat-balance equation (Yu and Rothrock, 1996; Drucker and others, 2003). The spatial resolution of MODISbased charts $h_{\mathrm{i}}^{\mathrm{M}}, \sim 1 \mathrm{~km}$, is much finer than that of the radiometer $h_{\mathrm{i}}$ charts. The major drawback with the $T_{\mathrm{s}}$-based $h_{\mathrm{i}}$ retrieval is the requirement for cloud-free conditions, which may introduce long temporal gaps in the $h_{\mathrm{i}}^{\mathrm{M}}$ chart. In addition, it is difficult to discriminate clear sky from clouds in winter night-time conditions (Frey and others, 2008).

Correlation between $h_{\mathrm{i}}$ and synthetic aperture radar (SAR) data has also been studied. For the Baltic Sea, Similä and others (2010) demonstrated that $h_{\mathrm{i}}$ estimation of deformed ice under dry snow conditions is possible through a statistical relationship between ice freeboard and radar backscatter. The variance of the mean freeboard (i.e. largescale surface roughness) increases with increasing mean freeboard, and, as the surface roughness increases, the backscatter also increases. Nakamura and others (2006) found good correlation between the $\mathrm{L}-(R=0.87)$ and Cband $(R=0.80)$ co-polarization ratio and $h_{\mathrm{i}}$ of undeformed ice in the Sea of Okhotsk. Good correlation has also been found between Antarctic first-year pack-ice and fast-ice thickness and the C-band co-polarization ratio (Nakamura and others, 2009). The co-polarization ratio has little sensitivity to ice surface roughness and is related to variations in salinity (i.e. ice surface dielectric constant) that can be caused by changes in $h_{\mathrm{i}}$ (Wakabayashi and others, 2004). Airborne C-band polarimetric SAR data together with a theoretical backscattering model have been used to estimate $h_{\mathrm{i}}$ in the $0-10 \mathrm{~cm}$ range (Kwok and others, 1995). The SAR-based methods for $h_{\mathrm{i}}$ retrieval are still experimental, and no operational products are available.

For the Baltic Sea, SAR images are used to produce a spatially more accurate level ice thickness chart (pixel size $500 \mathrm{~m} \times 500 \mathrm{~m})$ than the ice chart of the Finnish Ice Service (FIS) (resolution $10 \mathrm{~km}$; Karvonen and others, 2003, 2007). The ice area boundaries in the FIS ice chart are relocated to correspond to the area boundaries of the segmented, i.e. classified, SAR image. Inside the generated segments, thickness values are mapped to be between the minimum and maximum thickness values based on the SAR image segment backscattering mean values. The SAR-based level ice thickness chart shows the local mean ice thickness better than the FIS ice chart (Karvonen and others, 2004). In contrast to other SAR methods, an $h_{\mathrm{i}}$ chart is needed here as input, and SAR data are used to enhance its spatial resolution.

\section{DATASETS}

We tested our ice thickness method in winter 2008/09 over the Kara Sea. We acquired 32 coincident MODIS/Envisat ASAR WSM image pairs. AMSR-E radiometer data based sea-ice concentration (Spreen and others, 2008) at $6.25 \mathrm{~km}$ 
resolution (Polar View product) is used together with the HIGHTSI model to derive the background ice thickness field $H_{\text {b. }}$. The HIGHTSI model and its HIRLAM forcing data are described in detail in Cheng and others (2013).

The swath width of the WSM images is $406 \mathrm{~km}$, the image length is variable $(\sim 400 \mathrm{~km})$ and the pixel size is $75 \mathrm{~m}$. The polarization of the images is $\mathrm{HH}$. The incidence angle, $\Theta_{0}$, ranges from $16.3^{\circ}$ to $42.7^{\circ}$. The WSM images were rectified to a polar stereographic coordinate system with midlongitude of $63^{\circ} \mathrm{E}$ and true-scale latitude of $70^{\circ} \mathrm{N}$ using the European Space Agency (ESA)'s BEAM software. The pixel size of the rectified images is $100 \mathrm{~m}$, although the true resolution of a WSM image is $150 \mathrm{~m}$. The pixel values were converted to absolute $\sigma^{0}$ values. The equivalent number of looks (ENL) and noise equivalent $\sigma^{0}\left(\sigma_{N}^{0}\right)$ in the rectified WSM images were studied using WSM images over calm ocean. ENL is $\sim 18$ for the whole WSM $\Theta_{0}$ range. Thus, the radiometric resolution is $\sim 0.9 \mathrm{~dB} . \sigma^{0}{ }_{\mathrm{N}}$ depends on $\Theta_{0}$ : it is -24.5 to $-23 \mathrm{~dB}$ when $16.3 \leq \Theta_{0} \leq 25.9 ;-24.5$ to $-23 \mathrm{~dB}$ when $25.9 \leq \Theta_{0} \leq 39.2$; and -26 to $-24.5 \mathrm{~dB}$ when $\Theta_{0} \geq 39.2$. The absolute accuracy of $\sigma^{0}$ is $\pm 0.63 \mathrm{~dB}$ (ESA, 2009).

Due to the large variation of $\Theta_{0}$ in a single WSM scene, we performed a linear $\Theta_{0}$ correction proposed by Mäkynen and others (2002) with $32^{\circ}$ angle of incidence as the reference angle. Using their approach we determined an empirical correction factor of $-0.24 \mathrm{~dB}^{\circ-1}$ for the Kara Sea. This is very close to the factor of $-0.23 \mathrm{~dB}^{\circ-1}$ for the Baltic Sea determined by Mäkynen and others (2002).

The ice thickness $h_{\mathrm{i}}$ for thin-ice areas was retrieved from the MODIS-based $T_{\mathrm{s}}$ and modeled HIRLAM forcing data through the ice surface heat-balance equation ( $\mathrm{Yu}$ and Rothrock, 1996). This method and its uncertainty are described in detail in Mäkynen and others (2013). The maximum reliable MODIS-based ice thickness is $35-50 \mathrm{~cm}$ under typical polar winter weather conditions (air temperature below $-20^{\circ} \mathrm{C}$, wind speed $<5 \mathrm{~m} \mathrm{~s}^{-1}$ ). The uncertainty is lowest $(\sim 40 \%)$ for the $15-30 \mathrm{~cm}$ thickness range. We employed only night-time MODIS data. Thus, the uncertainties related to the effects of solar shortwave radiation and surface albedo were excluded. Despite several cloud tests and manual cloud masking, it was impossible to exclude all cloud-covered areas. For example, thin high clouds and ice fog are difficult to recognize even visually. Undetected high thin clouds result in a cold bias in $T_{\mathrm{s}}$, making the ice appear thicker than it actually is (Martin and others, 2004; Tamura and others, 2006). Ice fog generated by intense vapor from leads and polynyas under cold conditions is warmer than surrounding fast- or pack-ice $T_{\mathrm{S}}$ and colder than $T_{\mathrm{s}}$ for thin ice. This leads to underestimation of thickness for pack ice and overestimation for thin ice.

We use weekly AARI ice charts (Bushuev and Loshchilov, 2007) for the Kara Sea to validate our thickness charts. The AARI ice chart (available from http://wdc.aari.ru/datasets/ d0004/Kar/) shows the total ice concentration (in tenths) as well as concentration (in tenths), stage of ice development, ice thickness class and the form of ice (ice floe size) for the three thickest ice types for polygonal areas. The ice thickness is shown with World Meteorological Organization ice type nomenclature $(\mathrm{WMO}, 1989)$ (e.g. nilas $(<10 \mathrm{~cm})$, young ice $(20-30 \mathrm{~cm})$, first-year $(\mathrm{FY})$ thin ice $(30-70 \mathrm{~cm})$, FY medium ice $(70-120 \mathrm{~cm})$ and FY thick ice $(>120 \mathrm{~cm}))$. The ice charts are based on available visible and infrared satellite data, Envisat WSM images and reports from coastal stations and



Fig. 2. Overview of the multisensor and HIGHTSI sea-ice model based sea-ice thickness chart construction. On the left are the modeled data and their stepwise use. On the right are the used satellite datasets. The diagram indicates when and how the satellite data are used in deriving the thickness chart. IC is ice concentration.

ships. The segmentation of images and subsequent interpretation and mapping of ice conditions are carried out by ice experts. The AARI ice charts are in digital SIGRID format. The main purpose of the weekly ice chart is to show the spatial distribution and characteristics of sea ice. It is not intended for operational support of ice navigation. We have not found any publication that discusses the accuracy of the AARI weekly ice chart and how the degree of ice deformation (i.e. ice mass of the deformed ice fields) has been taken into account in the ice thickness typing.

\section{METHOD}

A flow chart presenting our ice thickness algorithm is shown in Figure 2. In this section we explain the different components of the method in detail.

\subsection{MODIS ice thickness chart}

The MODIS $h_{\mathrm{i}}$ chart is used to locate thin-ice areas (thickness $<40 \mathrm{~cm}$ ). When we compared the MODIS-based ice charts, $h_{\mathrm{i}}^{\mathrm{M}}$, to the AARI ice charts and to SAR imagery it seemed that sometimes $h_{\mathrm{i}}$ was underestimated. We also noticed that even if the obtained ice-thickness reconstruction is biased, it usually represents the ice field structure in a realistic manner when compared to SAR imagery. The major strength of the MODIS $h_{\mathrm{i}}$ retrieval is that it is directly based on sea-ice physics. The use of C-band SAR data in the seaice thickness estimation has more uncertainties because the relationship between $\sigma^{0}$ and ice type is ambiguous. We discuss this issue in Section 4.4.

\subsection{HIGHTSI ice thickness field}

We used the HIGHTSI model to create thermodynamically grown ice thickness field $H_{\mathrm{i}}$. For seasonal ice cover, thermodynamic processes dominate accretion and ablation of ice floes. Although HIGHTSI is a one-dimensional model, the spatial variation of snow and ice thickness can be obtained by applying HIGHTSI in a two-dimensional domain where the external forcing at each gridpoint comes from the HIRLAM model. In the HIGHTSI model, we used the same empirical snow vs ice thickness parameterization 
as in the MODIS-based $h_{\mathrm{i}}$ retrieval (Mäkynen and others, 2013). If precipitation from HIRLAM is used as input, we end up with snow that is too thick and, in consequence, ice cover that is too thin compared to the AARI charts. The empirical parameterization includes some effects of blowing-snow redistribution (e.g. some snow lost to open water areas), but using precipitation data does not.

In the HIRLAM data the grid size is $20 \mathrm{~km} \times 20 \mathrm{~km}$. At each gridpoint, the ice/no-ice condition was determined by the AMSR-E-based sea-ice concentration chart. Areas of ice concentration less than 15\% were considered open water. In the model run, the occurrence of open water in a previously ice-covered area was also taken into account: the model run was stopped when the gridpoint was situated in open water, and the previous snow and ice parameters were resumed when this gridpoint was ice-covered again. This gives reasonable results when we have a practically closed basin where the majority of pack ice moves back and forth from the nearby gridpoints (e.g. in the semi-enclosed Bay of Bothnia in the Baltic Sea). In the Kara Sea, where the ice conditions are highly dynamic and the basin has a large open boundary, this approach produced both under- and overestimates of ice thickness in different conditions.

The static $H_{\mathrm{i}}$ field yielded $h_{\mathrm{i}}$ values that were too large in several instances. To gain insight into these errors, their magnitude and spatial location, we compared the weekly AARI ice charts to the modeled $H_{\mathrm{i}}$ field. Overestimation typically occurs where the ice edge has retreated and new ice is formed in opened sea. Thus the activation of the HIGHTSI model run with previous ice and snow parameters produces ice that is too thick. The same applies to large polynyas. In the Kara Sea during December and January the $H_{\mathrm{i}}$ values were $0-40 \mathrm{~cm}$ thicker compared to the AARI ice charts. In the southern Kara Sea the difference was especially significant. Modeled $H_{\mathrm{i}}$ values that were too large persisted through February and March in the southern Kara Sea and, naturally, in polynyas in the whole Kara Sea. The difference between $H_{i}$ values and the dominant mean thickness according to the AARI ice charts began to reduce in February in the northern Kara Sea basin. The $H_{\mathrm{i}}$ values were in agreement with, or slightly greater than, mean AARI ice-chart ice thickness values only in the central Kara Sea during February and March. From mid-March the $H_{\mathrm{i}}$ values could be considered to be generally close to those of the AARI charts. In April the trend slightly reversed so that the $H_{\mathrm{i}}$ values often stayed below those in the AARI charts. However, the overestimation bias through a period of 3.5 months affected our utilization of $H_{\mathrm{i}}$ values because the majority of our images (30 out of 32) were acquired during that period.

\subsection{Background ice thickness field}

In order to introduce large-scale ice dynamics into $H_{i}$, we used changes in the daily AMSR-E ice concentration charts. We recorded the ice concentration history in the test area during the 14 days prior to the ice chart construction day to modify the thermodynamic ice thickness field. A set of rules was created to extract information on mesoscale ice dynamics and occurrences of large polynyas from the ice concentration history. The goal of these rules is to locate significant divergent events. We reduced $H_{\mathrm{i}}$ in the areas where these events had occurred.

Based on the ice concentration statistics, we constructed a matrix $W$ which was applied to the $H_{\mathrm{i}}$ field to obtain a modified background ice thickness field $H_{\mathrm{b}}$ :

$$
H_{\mathrm{b}}=W H_{\mathrm{i}} \text {. }
$$

$H_{\mathrm{b}}$ is then used in the SAR classification. The value of an element $w$ in $W$ depends on the recent local ice concentration history, the emphasis being on the $0-5$ days prior to the analysis day. The $W$ matrix tells us the locations of those areas where a large-scale divergent event (minimum diameter of area: $10-20 \mathrm{~km}$ ) has recently taken place. Derivation of the $w$ coefficients is discussed in detail in the Appendix.

The matrix $W$ describes the progress of the freeze-up period well because, due to the thin ice cover in early winter, ice edge and ice concentration undergo rapid changes. In the Kara Sea the freeze-up period lasted until early February in winter 2008/09. Even after the freeze-up the occurrence frequency of new polynyas was high. The spatial distribution of small $w$ values corresponded well with the thin-ice locations. This was verified by comparing $W$ to the MODIS charts $h_{\mathrm{i}}^{\mathrm{M}}$ and SAR imagery. Leads, however, are too narrow to be detected by the AMSR-E data.

The accuracy and the information value of $W$ also depends on ice drift. Are the thin-ice areas where $W$ predicts them to be if the ice concentration chart of the analysis day shows $100 \%$ ice concentration for the areas in question? The spatial distribution of $w$ consists mostly of features with diameter of 30-60 km (5-10 AMSR-E pixels). Most of the $w$ values are determined on the basis of the $0-5$ days prior to the analysis. For a substantial overlap between the thin-ice areas indicated by $H_{\mathrm{b}}$ and the ice conditions on the analysis day, the ice displacement should not exceed $30 \mathrm{~km}$ in order that the large-scale $H_{\mathrm{b}}$ features $(30-60 \mathrm{~km})$ cover approximately the areas determined by $W$. Similarly, the ice displacement should not exceed $20 \mathrm{~km}$ if we want the location of the smaller-scale $H_{\mathrm{b}}$ features to have reasonable accuracy. Our own SAR-based ice-drift estimates for a typical ice displacement in the Kara Sea in the closed ice pack were $3-10 \mathrm{~km} \mathrm{~d}^{-1}$ (cf. Leppäranta, 2005). Considering the zigzag character of ice drift and typical ice displacement values in the test area, the thin-ice areas at the analysis day mostly overlap with areas indicated by $W$. Comparison with the MODIS charts $h_{\mathrm{i}}^{\mathrm{M}}$ supports this conclusion.

The $W$ matrix is a rough approximation for the ice dynamics on the mesoscale. A fundamental limitation in the use of the $W$ matrix is that it is not able to indicate convergence. All information related to the ice deformation must be derived from SAR $\sigma^{0}$.

\subsection{SAR-based sea-ice classification}

Here we address the problem of C-band $\sigma^{0}$-based sea-ice classification. When the incidence angle is $<45^{\circ}$, the backscattering at $\mathrm{C}$-band $\mathrm{HH}$-polarization is dominated by the ice surface scattering, for the Arctic sea ice (Onstott, 1992) and Baltic sea ice (Carlström and Ulander, 1995; Dierking and others, 1999). This implies that the magnitude of $\sigma^{0}$ is modulated mainly by the small-scale ( $\mathrm{mm}$ to $\mathrm{cm}$ ) and large-scale ( $\mathrm{cm}$ to $\mathrm{m}$ ) surface roughness. The $\sigma^{0}$ of level ice can increase to that of deformed ice (Manninen, 1996) but this rarely happens. In most cases, $\sigma^{0}$ of level ice is smaller than that of deformed ice.

In an extensive recent study, Zakhvatkina and others (in press) determined $\sigma^{0}$ ranges for different ice types in the central Arctic (four first-year ice (FYI) types and multi-year ice (MYI) category). They used Envisat WSM images and 
mean $\sigma^{0}$ over an area of $1.1 \times 1.1 . \mathrm{km}^{2}$. The ice type determination was based on visual interpretation of the SAR data. Their results concerning ice types are close to those obtained by Similä and others (2010) in the Baltic Sea, and also for Envisat SAR images where the three-dimensional (3-D) ice freeboard topography constructed from the laser scanner measurements acted as the validation data. It was also noted in these studies that good accuracy detection of ice categories based only on the $\sigma^{0}$ value is not feasible. The encouraging result of both studies was that the major FYI categories (nilas, young ice, level ice and deformed ice) can be roughly extracted from SAR data relying on the $\sigma^{0}$ values. The classification results must then be refined with textural features and careful statistical modeling and/or using sea-ice models.

It is well known that the average $\sigma^{0}$ increases when the FYI deformation increases, due to the dominance of surface scattering at the C-band (Onstott, 1992; Kwok and Cunningham, 1994; Lundhaug, 2002). However, the relationship between $h_{\mathrm{i}}$ and $\sigma^{0}$ is complicated because the ice deformation seen in SAR imagery is not always directly related to $h_{\mathrm{i}}$. Large-scale surface roughness appears in many shapes and forms (e.g. brash ice, pancake ice, rafted ice, hummocked and ridged ice). These FYI types have widely differing $h_{\mathrm{i}}$ categories, from 5-15 cm (pancake ice) to several meters (heavily ridged areas). All these ice types typically have large $\sigma^{0}$ values. The interpretation of $\sigma^{0}$ is further complicated by the dependence of $\sigma^{0}$ on the incidence angle and other properties of SAR imagery (e.g. noise equivalent backscattering coefficient $\sigma_{N}^{0}$ ).

Some of the variability of the Envisat SAR $\sigma^{0}$ signatures can be seen in Figure 1, with probability density functions for each ice type. The ice cover was divided into four ice types: nilas $(<10 \mathrm{~cm})$, young ice $(10-30 \mathrm{~cm})$, smooth FYI (totally or mostly undeformed ice) and rough FYI pack ice where visible traces of deformation were detected. The first two ice types were identified using MODIS $h_{i}{ }^{\mathrm{M}}$, and the last two using SAR imagery. The $\sigma^{0}$ values are mean values calculated over a $3.1 \times 3.1 \mathrm{~km}^{2}$ area.

In order to translate the magnitude of $\sigma^{0}$ into a specific ice type in a meaningful manner, we use the results shown in Figure 1, the results presented in the literature and the experience gained by visually interpreting a multitude of SAR images over the Kara Sea. We averaged the $\sigma^{0}$ values over a $2 \times 2 \mathrm{~km}^{2}$ area which made the effect of speckle negligible. An additional advantage is that an area of $4 \mathrm{~km}^{2}$ represents an ice type better than a single pixel with 100 $400 \mathrm{~m}$ resolution. On the other hand, in an area of $4 \mathrm{~km}^{2}$, ice is often a mixture of several ice types.

In our SAR images the dynamic $\sigma^{0}$ range at $400 \mathrm{~m}$ resolution was from $-20 \mathrm{~dB}$ (smooth new ice) to $-9 \mathrm{~dB}$ (pancake ice). Larger $\sigma^{0}$ values also appeared but they originated mostly from open water. The effective dynamic $\sigma^{0}$ range at $2 \mathrm{~km}$ resolution is smaller and rather narrow, most of the pack-ice $\sigma^{0}$ values being concentrated in the interval $[-16 \mathrm{~dB},-12 \mathrm{~dB}]$. The $\sigma^{0}$ values outside this interval usually came from open water or different thin-ice types (e.g. pancake ice).

Table 1 shows our $\sigma^{0}$-based sea-ice type classification. The terms heavily and slightly deformed ice fields refer to the areal fraction of deformed ice. The $h_{\mathrm{i}}$ difference for the ice types 'mostly level ice' and 'smooth level ice' is small. The latter ice type refers to a newer level ice with smaller surface roughness and smaller $h_{\mathrm{i}}$. Some of the $h_{\mathrm{i}}$ values for thin ice
Table 1. The $\sigma^{0}$ limits and the values of the factor $F$ used in the construction of the sea-ice thickness chart $h_{\mathrm{ch}}$. The backscattering coefficient $\sigma^{0}$ is averaged over SAR data of $2 \mathrm{~km} \times 2 \mathrm{~km}$. The applied $\sigma^{0}$ limits for the sea-ice types are based on the literature and experiments. The scaling factor $F$ is explained in Section 4.4. The ice thickness chart $h_{\mathrm{ch}}$ is defined in Eqn (3)

\begin{tabular}{|c|c|c|c|}
\hline$\sigma^{0}$ range & Ice type & $F$ & $\begin{array}{l}\text { Effective } h_{\text {ch }} \\
\quad \text { range }\end{array}$ \\
\hline $\mathrm{dB}$ & & & $\mathrm{m}$ \\
\hline$>-9$ & Open water & 0 & 0 \\
\hline$[-10,-9]$ & $\begin{array}{l}\text { Brash ice, pancake ice } \\
\text { open lead }\end{array}$ & 0 & 0.2 \\
\hline$[-11.25,-10]$ & Broken thin ice & 0 & 0.3 \\
\hline$[-13,-11.25]$ & $\begin{array}{l}\text { Heavily deformed ice } \\
\text { (ridges, hummocked ice) }\end{array}$ & 1 & $0.3-1.2$ \\
\hline$[-14.5,-13]$ & $\begin{array}{l}\text { Slightly deformed ice } \\
\text { (ridges, hummocked ice) }\end{array}$ & 0.8 & $0.3-0.95$ \\
\hline$[-15,-14.5]$ & $\begin{array}{l}\text { Mostly level ice } \\
\text { minor deformation possible }\end{array}$ & 0.6 & $0.3-0.7$ \\
\hline$[-16.5,-15.0]$ & $\begin{array}{c}\text { Smooth level ice } \\
\text { minor deformation possible }\end{array}$ & 0.5 & $0.3-0.6$ \\
\hline$<-16.5$ & Smooth thin ice & 0 & 0.2 \\
\hline
\end{tabular}

are due to the rules based on the ice concentration history as detailed in the Appendix.

The ice thickness chart estimates, $h_{\mathrm{ch}}$, are now determined according to the $\sigma^{0}$ and $H_{\mathrm{b}}$ values. We define $h_{\mathrm{ch}}$ as

$$
h_{\mathrm{ch}}=F H_{\mathrm{b}}
$$

where the scaling factor $F$ modifies the ice thickness field $H_{\mathrm{b}}$. We let $F$ depend only on the magnitude of $\sigma^{0}$. As discussed in Section 4.2, $H_{\mathrm{b}}$ is the upper limit for the thermodynamically grown ice, often even exceeding it. Here the range of $F$ is $0.5-1$, and in some cases 0 . If we had frequently updated information about local deformation rates, the value of $F$ could be $>1$. The current information about the local ice deformation is so uncertain that we conservatively choose 1 as the upper limit for $F$. We simplified the $\sigma^{0}$-based sea-ice classification by letting $F$ and, in consequence, sea-ice thickness gradually increase when $\sigma^{0}$ increases to saturation point (here $-11.25 \mathrm{~dB}$ at the $32^{\circ}$ incidence angle). The effective ranges of $h_{\mathrm{ch}}$ were derived from the range of $H_{\mathrm{b}}$, which had a maximum value of $120 \mathrm{~cm}$ in the drift ice according to the HIGHTSI model during the analysis period. The first analyzed SAR/MODIS image pair was acquired on 5 December 2008, and the last image pair on 21 April 2009.

When we assigned an ice type and an $F$ value in Table 1 to a specific $\sigma^{0}$ range, we did it knowing the ambiguous relationship between $\sigma^{0}$ and ice type. In Table 1 we have chosen, for a given $\sigma^{0}$ range, the most likely ice type based on our knowledge (e.g. Fig. 1 and subjective experience). We also utilized our earlier results (Similä and others, 2010), which are based on a field dataset, as guidance for a geophysically reasonable sea-ice classification. We recall that the results of Zakhvatkina and others (in press) for the central Arctic are in good agreement with those of Similä and others (2010) obtained in the Baltic Sea. The $\sigma^{0}$ values in Table 1 deviate slightly from those in Figure 1 . The reasons for this are the different pixel sizes in our sea-ice classification and data in Figure 1 (2 km vs $3.1 \mathrm{~km}$ ) as well as our experiences when we varied the class boundaries. We 


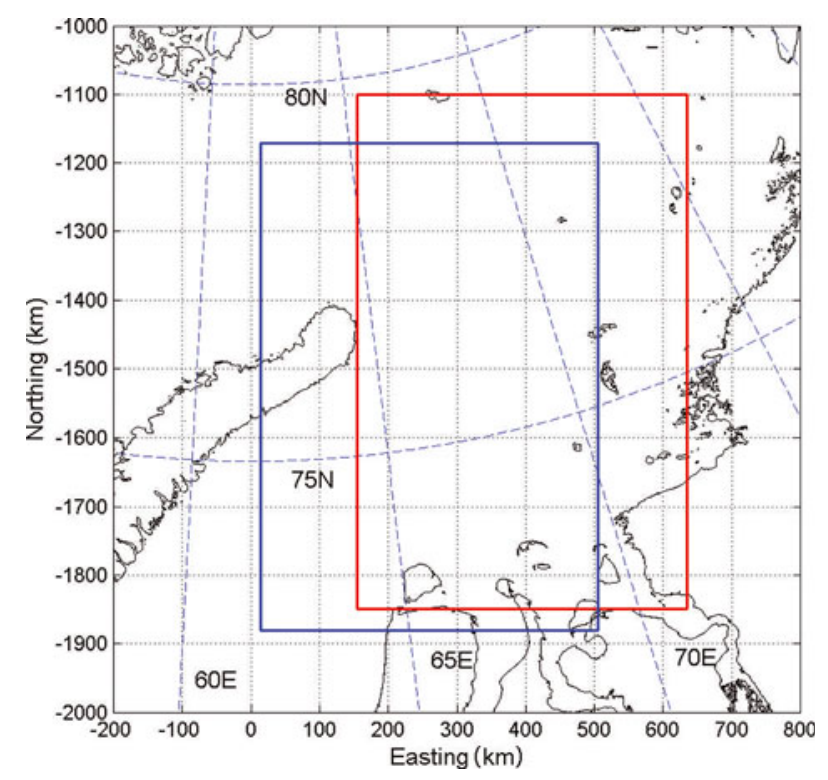

Fig. 3. The locations of the ice thickness charts for 4 March 2009 (blue rectangle) and 7 February 2009 (red rectangle) in the Kara Sea.

lacked field data to quantify the accuracy of the proposed SAR-based sea-ice classification. However, we are able to assess the uncertainty of the $h_{\mathrm{ch}}$ chart, to some extent, in Section 6.

\subsection{Ice thickness chart}

To produce the overall ice thickness chart, we combine the different data sources at our disposal. Over cloud-free areas, thin-ice regions ( $h_{\mathrm{i}}$ up to $40 \mathrm{~cm}$ ) are identified on the basis of MODIS data, and over cloud-covered areas they are located using the matrix $W$.

The overall (HIGHTSI/AMSR-E/MODIS/SAR) ice thickness chart is constructed as follows:

$$
\begin{aligned}
& h_{\mathrm{ch}}=h_{\mathrm{i}}^{\mathrm{M}}, \text { if } h_{\mathrm{i}}^{\mathrm{M}} \text { defined and } h_{\mathrm{i}}^{\mathrm{M}} \leq 0.4 \mathrm{~m}, \\
& h_{\mathrm{ch}}=F H_{\mathrm{b}}, \text { if } h_{\mathrm{i}}^{\mathrm{M}}>0.4 \mathrm{~m} \text { or } h_{\mathrm{i}}^{\mathrm{M}} \text { undefined. }
\end{aligned}
$$

We required a significant fraction of the MODIS $h_{\mathrm{i}}^{\mathrm{M}}$ to be cloud-free to increase the reliability of the cloud detection and, in consequence, the MODIS-based $h_{\mathrm{i}}$ estimates. The detection of thin-ice areas using the $W$ matrix succeeded only partially and only for large areas (diameter of area: $>10-20 \mathrm{~km})$. The construction of $h_{\mathrm{ch}}$ necessitated that the MODIS/SAR image pairs were acquired almost simultaneously due to ice drift. The cloud-free requirement for $h_{i}^{M}$ prevented us from calculating the $h_{\text {ch }}$ chart regularly for a given area. The infrequent availability of the cloud-free MODIS images made it impossible to track ice motion using the AMSR-E/MODIS/SAR datasets.

The $h_{\text {ch }}$ charts do not provide proper $h_{\mathrm{i}}$ estimates for fastice areas. For the MODIS $h_{\mathrm{i}}^{\mathrm{M}}$ chart, the main problem is that the thickness of fast ice exceeds the $h_{\mathrm{i}}$ retrieval threshold of $40 \mathrm{~cm}$ quite early in the winter. Another complicating factor is the relatively thick snow layer on fast ice. In winter 2008/ 09 the landfast ice reached a thickness of $0.45-0.5 \mathrm{~m}$ in the coastal area of the northern Kara Sea in late November. In the southern Kara Sea it exceeded $0.5 \mathrm{~m}$ thickness in midDecember in several coastal areas (Cheng and others, 2013).

For the ice concentration charts from AMSR-E radiometer data, the pixels near the coast are contaminated by the land spill effect. The land-contaminated radiometer data have been estimated to extend about three times the spatial resolution of the data from the coast (Maaß and Kaleschke, 2010). This is $\sim 37.5 \mathrm{~km}$ for the ice concentration data used here. Using an ice-motion tracking algorithm for SAR images, one can in principle delineate the fast-ice boundaries at any given time. This task was not performed in our study.

\section{APPLICATIONS}

Before we present the accuracy analysis for our $h_{\mathrm{ch}}$ charts, we demonstrate the ice thickness algorithm with two examples. The locations of the two sample images are shown in Figure 3.

There are, to our knowledge, very few English-language studies on sea-ice thickness in the Kara Sea. The most comprehensive overview of ice conditions in the Kara Sea is given in Johannessen and others (2007). Their information on $h_{\mathrm{i}}$ is climatological in nature. Based on electromagnetic measurements in the southern Kara Sea, Haas and others (1999) reported typical $h_{\mathrm{i}}$ ranges of $1-1.5 \mathrm{~m}$ for pack-ice fields in April/May 1998. In our $h_{\text {ch }}$ charts, typical values from early to mid-April ranged from 0.7 to $1.2 \mathrm{~m}$ in the same area. Throughout April 2009 the ice cover thickened according to the AARI ice charts. Winter 2008/09 was, however, much milder than winter 1997/98. In winter 1997/ 98 the Kara Sea was ice-covered at the end of November. In winter 2008/09 this happened in late January, i.e. almost 2 months later. Divine and others (2005) have studied fast-ice variability and its connection to atmospheric forcing in the northeastern Kara Sea.

First we examine ice conditions on 4 March 2009 where we have a coincident SAR/MODIS image pair and an AARI ice chart (Figs 4 and 5). The weather was cold: air temperature $T_{\text {a }}$ was $-27^{\circ} \mathrm{C}$. In the AARI ice chart we see a narrow polynya near Novaya Zemlya, and that FYI thin ice $(<70 \mathrm{~cm})$ was the dominant ice type on the western side of the Yamal peninsula and north of it along the coast up to Severnaya Zemlya. The polynyas can be detected from the SAR image. In the MODIS chart, $h_{\mathrm{i}}{ }^{\mathrm{M}}$, the polynyas are only weakly visible, although the thin-ice areas $(<30 \mathrm{~cm})$ near the ice edge at the northern tip of Novaya Zemlya are identified well. We observe that the matrix $W$ captured part of the FY thin-ice areas in addition to the very thin ice fields near the ice edge. There were errors in $W$ in the fast-ice area located northeast of the Ob river estuary. In the overall thickness chart, $h_{\mathrm{ch}}$, the major features are present concerning the spatial distribution of thin $\left(h_{\mathrm{ch}}<70 \mathrm{~cm}\right)$ and thick ice $(>70 \mathrm{~cm})$ areas (Fig. 5).

For comparison we also include the SAR/MODIS thickness chart which is the same chart as $h_{\text {ch }}$ except that no thickness estimates are given to the cloud-covered region. We notice that the thin-ice area at the northern tip of Novaya Zemlya is retrieved well. We also observe that some thin-ice zones marked in the AARI chart and visible in the SAR image are detected in the northern part of $h_{\mathrm{ch}}$ which is masked off from the SAR/MODIS thickness chart.

The second $h_{\text {ch }}$ chart shown here was constructed for 7 February 2009 (Figs 6 and 7). The AARI ice chart shows ice conditions 3 days earlier, on 4 February 2009. During the intervening 3 days the weather was cold. $T_{\text {a }}$ stayed well below $-30^{\circ} \mathrm{C}$. According to the SAR imagery and the AARI ice chart, several very thin ice areas appeared in the northeastern part of the Kara Sea. These thin ice areas were detected well in the $h_{\mathrm{ch}}$ chart, where they were identified on the basis of MODIS chart $h_{\mathrm{i}}^{\mathrm{M}}$. The western part of $h_{\mathrm{ch}}$ shows 

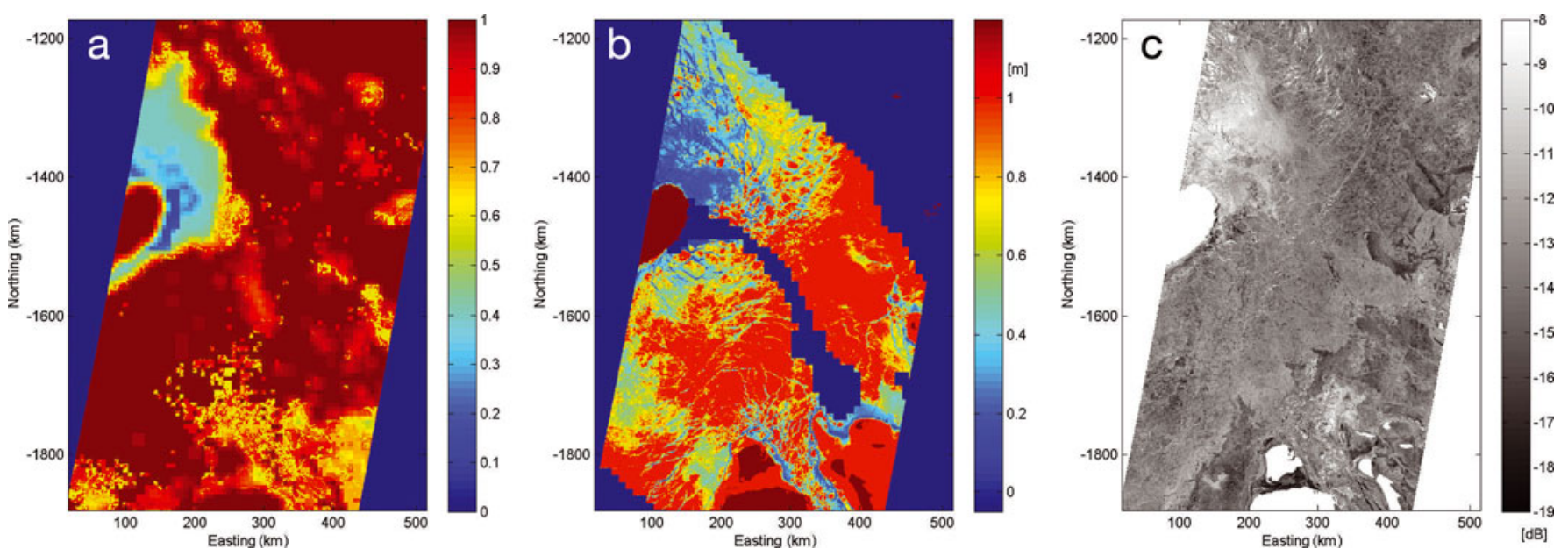

Fig. 4. Datasets used in constructing the ice thickness chart on 4 March 2009. (a) The ice concentration (IC)-based weight matrix $W$; (b) the MODIS-based $h_{\mathrm{i}}$ (dark blue shows no-data and cloudy areas; $h_{\mathrm{i}}=1 \mathrm{~m}$ signifies $h_{\mathrm{i}} \geq 1 \mathrm{~m}$ ); and (c) the Envisat ASAR WSM image. All panels are shown in polar stereographic coordinates with reference longitude $63^{\circ} \mathrm{E}$. Land area is marked dark brown in (a, b) and white in (c).

thinner ice than in the AARI chart. This was probably due to undetected warm ice fog in the MODIS ice surface temperature. The borderline in the north-south direction separating thicker $\left(h_{\mathrm{ch}}\right.$ mostly $\left.>70 \mathrm{~cm}\right)$ and thinner ice areas refers to an artifact in the MODIS chart $h_{\mathrm{i}}^{\mathrm{M}}$ (Fig. 6b).

The ice thickness for the cloud-covered area in the $h_{\mathrm{ch}}$ chart (Fig. 7a) matched the AARI ice chart with the exception of the southeastern corner of the SAR image. Based on visual inspection of the SAR image, this area was covered by a mixture of open water and brash ice. In the $h_{\mathrm{ch}}$ chart it was estimated to have significantly thicker ice.

In our $32 \mathrm{MODIS} / \mathrm{SAR}$ image pair data, in $>20 \%$ of the pairs the MODIS charts, $h_{\mathrm{i}}{ }^{\mathrm{M}}$, contained anomalies contradicting the information in the SAR imagery. If the $h_{\mathrm{ch}}$ charts are used more extensively, the resulting $h_{\mathrm{ch}}$ must be endowed with a quality flag indicating the reliability of the estimated $h_{\mathrm{i}}$. The quality flag could be based on the human assessment of the result. It may be possible to automate the quality control process.

\section{ACCURACY ASSESSMENT OF THE ICE THICKNESS CHART}

The accuracy of the $h_{\mathrm{ch}}$ charts was estimated by comparing them to the AARI ice charts. The absolute accuracy of the AARI ice charts using satellite data has not been established (personal communication from V. Smolyanitsky, 2013). To reduce the effect of ice drift we selected the $h_{\mathrm{ch}}$ charts that were computed within 1 day of the weekly AARI ice chart. This requirement was met seven times. Five $h_{\mathrm{ch}}$ charts used in the validation are for March (on 4, 10, 12, 18 and 25 March), one for December (25 December) and one for February (10 February).

From the AARI ice chart we can extract the three most dominant ice types and their relative concentrations for each polygon. For the seven selected AARI ice charts these ice types were typically nilas $\left(h_{\mathrm{i}}<10 \mathrm{~cm}\right)$, young ice $\left(h_{\mathrm{i}}=\right.$ $10-30 \mathrm{~cm}), \mathrm{FYI}$ thin ice $\left(h_{\mathrm{i}}=30-70 \mathrm{~cm}\right)$ and FYI medium ice $\left(h_{\mathrm{i}}=70-120 \mathrm{~cm}\right)$. In the accuracy analysis we simplified
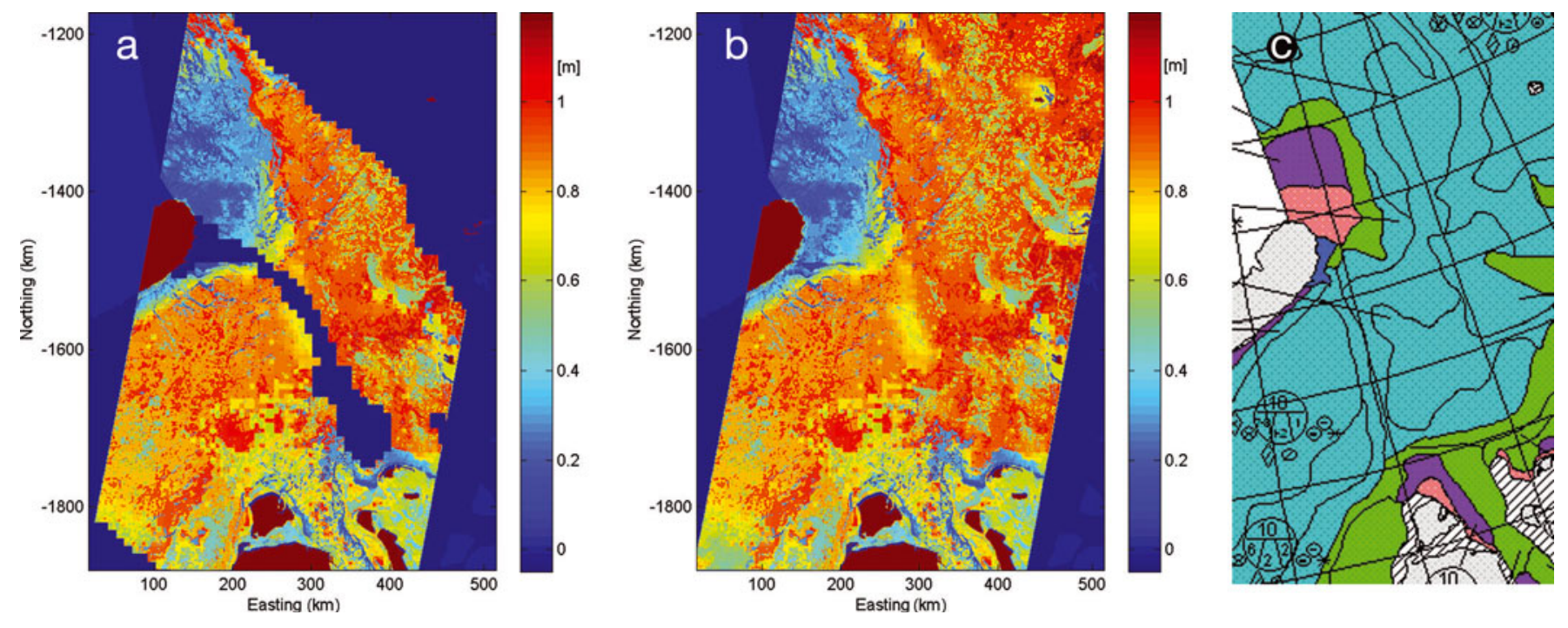

Fig. 5. The ice thickness charts on 4 March 2009. (a, b) The MODIS/SAR ice thickness chart (a) and the ASMR-E/MODIS/SAR thickness chart (b) (both charts in polar stereographic coordinates). The dark blue areas with $h_{\mathrm{i}}=-0.1$ represent areas with no data, i.e. they are outside the SAR image or clouded regions (a). Land is marked dark brown. (c) The AARI ice chart on 4 March 2009, with color codes green = mainly thin FYI $(30-70 \mathrm{~cm})$, light blue = mainly medium FYI $(70-120 \mathrm{~cm})$, the rest being thin ice types $(0-30 \mathrm{~cm})$. 

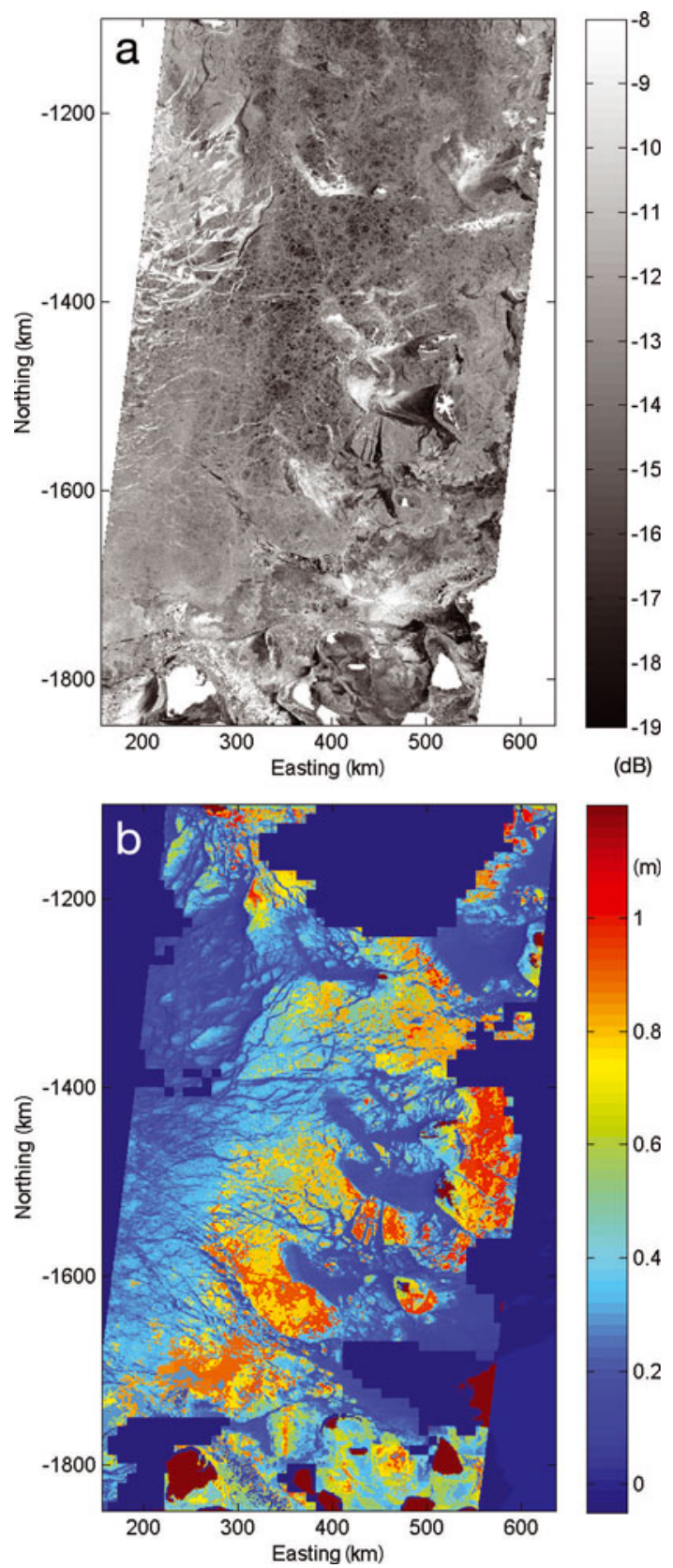

Fig. 6. (a) Envisat ASAR WSM image acquired on 7 February 2009. (b) MODIS/SAR ice thickness chart where the cloud-covered area is marked dark blue $\left(h_{\mathrm{i}}=-0.1\right)$. The value $h_{\mathrm{i}}=-0.1$ is also used also to represent areas with no data. Both panels are shown in the same coordinate system as in Figure 4. Land (mostly islands) is marked white in (a) and dark brown in (b).

the situation slightly and used only three ice categories: very thin ice $\left(h_{\mathrm{i}}=1-30 \mathrm{~cm}\right), \mathrm{FYI}$ thin ice and FYI medium ice. Small open water areas were included in the thin ice category.

The validation of our $h_{\mathrm{ch}}$ charts is based on the relative frequencies of the three ice classes mentioned above. In the seven AARI ice charts, there are altogether 84 polygons which overlapped our $h_{\mathrm{ch}}$ charts. Each of these polygons has its own ice-class frequency distributions. Very small polygons $\left(<20 \mathrm{~km}^{2} ; 25 \%\right.$ of all polygons $)$ are studied separately.

We applied two different statistics to measure the closeness of the relative frequencies originating from the AARI ice charts and from our $h_{\text {ch }}$ charts. The first statistic is
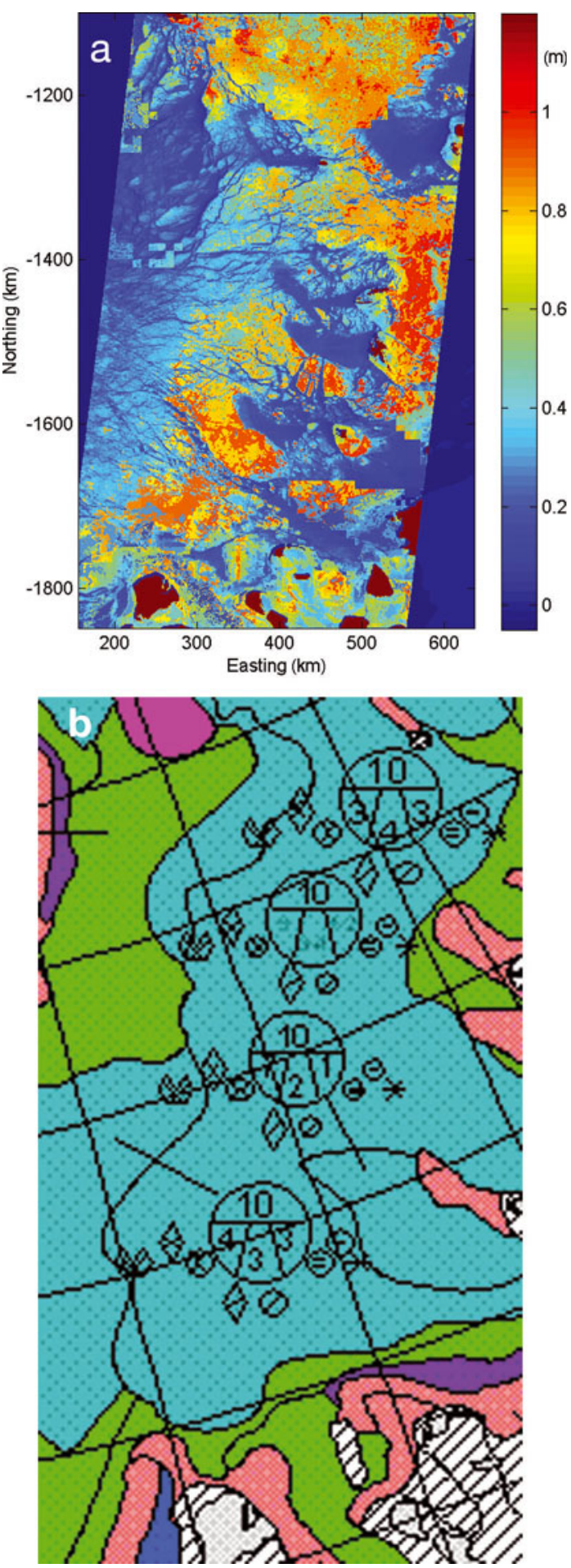

Fig. 7. (a) HIGHTSI/AMSR-E/MODIS/SAR ice thickness chart $h_{\mathrm{ch}}$ for 7 February 2009. (b) AARI ice chart on 4 February 2009, with color codes green $=$ mainly thin $\mathrm{FYI}(30-70 \mathrm{~cm})$, light blue $=$ mainly medium FYI $(70-120 \mathrm{~cm})$, the rest being thin ice types $(0-30 \mathrm{~cm})$. The dark blue areas in (a) $\left(h_{\mathrm{i}}=-0.1\right)$ represent areas with no data. Land area is marked dark brown.

the approximate mean ice thickness, $h_{x,}$ of the polygon derived from the ice type frequencies:

$$
h_{x}[\mathrm{~cm}]=15 \operatorname{freq}_{x}\left(c_{1}\right)+50 \operatorname{freq}_{x}\left(c_{2}\right)+95 \operatorname{freq}_{x}\left(c_{3}\right) \text {, }
$$

where the subscript $x$ refers to the dataset from which the class-wise frequencies freq $_{x}$ originated, a for the AARI chart and $e$ for $h_{\mathrm{ch}}$. freq ${ }_{x}\left(c_{k}\right)$ refers to the relative frequency of the ice type $c_{k}$ in the polygon. We selected the 


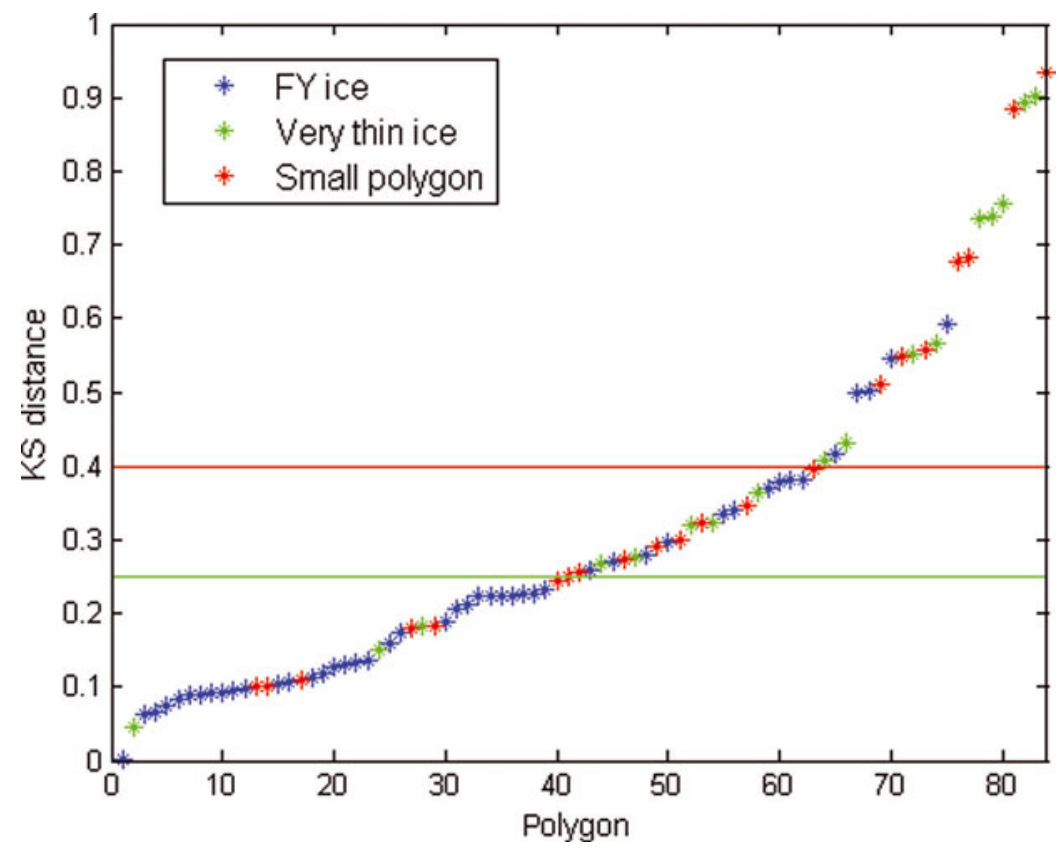

Fig. 8. The Kolmogorov-Smirnov distance for the AARI ice chart polygons used in the $h_{\mathrm{ch}}$ validation. For every polygon the KS distance was calculated between the cumulative frequency functions extracted from the $h_{\mathrm{ch}}$ chart and the AARI ice chart, respectively. Very thin ice polygons (mean thickness $<30 \mathrm{~cm}$ ) and small polygons $\left(\right.$ area $<20 \mathrm{~km}^{2}$ ) are marked green and and red. If the KS statistic lies below the green line, the class-wise frequency distributions of the the AARI chart and $h_{\mathrm{ch}}$ are considered consistent. If it is above the red line, the cumulative frequency distributions are inconsistent.

midpoint of the thickness range of an ice type to represent its typical thickness.

For validation purposes we divided the polygons into ice categories according to the mean thickness $h_{a}$, i.e. if $h_{a}$ was $<30 \mathrm{~cm}$, it was labeled $c_{1}{ }^{a}$, etc. Using this criterion, the polygons belonged in $22(26 \%)$ cases to the very thin ice category, in $41(49 \%)$ cases to the FYI thin ice category and in $21(25 \%)$ cases to the FYI medium ice category. In the same manner, we labeled the polygons based on $h_{\mathrm{e}}$ thickness. Because the thickness for each ice category was fixed, the label of a polygon depended only on the relative frequencies of the three ice types. This enables us to study the agreement of the frequency distributions on the basis of these two different polygon labelings. The comparison is presented in Table 2. Each row shows the number of polygons of same ice type category according to $h_{\mathrm{a}}$ (AARI charts). The columns shows the ice type categories for these polygons according to $h_{\mathrm{e}}$. In the diagonal is the number of polygons where estimated and AARI-based ice thickness categories were in agreement. According to the error matrix shown in Table 2, the estimated ice type and the AARI-based ice type agreed in only $24 \%$ of cases for the very thin ice category. The results were better for the FYI thin ice category (agreement in $85 \%$ of cases) and the FYI medium ice category (agreement in $76 \%$ of cases).

The second, and a more common, statistic to measure the closeness of two frequency distributions is a discrete variant of the Kolmogorov-Smirnov (KS) statistic (Dudewicz and Mishra, 1988). We calculate it as

$$
\mathrm{KS}=\max _{k}\left|F_{\mathrm{e}}\left(c_{k}\right)-F_{\mathrm{a}}\left(c_{k}\right)\right|, \text { where } k \in\{1,2,3\},
$$

where $F_{\mathrm{e}}$ and $F_{\mathrm{a}}$ denote the cumulative frequency functions of the $h_{\mathrm{ch}}$ chart and the AARI ice chart, respectively. The symbol $c_{k}$ refers to the $k$ th ice category.

The KS-statistic values for the polygons are shown in Figure 8. We chose the thresholds for the KS values subjectively. The chosen values were relatively large because there were only three classes, i.e. three jumps in the cumulative frequency function. We assessed that if the $\mathrm{KS}$ values stayed below 0.25 , there was good consistency between $F_{\mathrm{e}}$ and $F_{\mathrm{a}}$. Similarly we chose 0.4 to signify a threshold after which $F_{\mathrm{e}}$ and $F_{\mathrm{a}}$ resembled each other insignificantly.

In $49 \%$ of cases, $F_{\mathrm{e}}$ was in good agreement with $F_{\mathrm{a}}$. The agreement was poor or non-existent in $25 \%$ of cases. When studying the poor agreement polygons, we found that most of them were either small polygons $(33 \%$ of poor agreement cases) or represented very thin ice $(61 \%$ of poor agreement cases). Few poor agreement polygons were small and represented thin ice. The ice categories of the polygons were based on the $h_{\mathrm{a}}$ values as above. Of the small polygons, one-third stayed below the 0.25 threshold. The detection of very thin ice class was not successful. Only $19 \%$ of these polygons stayed below the 0.25 limit. The results for the polygons representing ice types FYI thin or FYI

Table 2. The error matrix obtained in the validation for 84 polygons (see Section 6). The table shows the comparison between the AARI ice chart based ice types $\left(c_{k}{ }^{\mathrm{a}}\right)$ vs ice thickness chart ice types $\left(c_{k}{ }^{\mathrm{e}}\right)$. The ice types are: very thin ice $\left(c_{1}{ }^{x}\right), F Y$ thin ice $\left(c_{2}{ }^{x}\right)$ and FY medium ice $\mathrm{c}_{3}{ }^{x}$. The subscript $x$ indicates the data source, $x=a$ for the AARI charts, $x=e$ for the $h_{\mathrm{ch}}$ charts. The polygons were divided into ice categories according to the mean thicknesses $h_{\mathrm{a}}$ and $h_{\mathrm{e}}$ (see Eqn (4))

\begin{tabular}{llrr}
\hline Ice type & $\mathrm{C}_{1}{ }^{\mathrm{e}}$ & $\mathrm{C}_{2}{ }^{\mathrm{e}}$ & $\mathrm{C}_{3}{ }^{\mathrm{e}}$ \\
\hline $\mathrm{C}_{1}{ }^{\mathrm{a}}$ & 5 & 16 & 1 \\
$\mathrm{C}_{2}{ }^{\mathrm{a}}$ & 1 & 35 & 5 \\
$\mathrm{C}_{3}{ }^{a}$ & 0 & 5 & 16 \\
\hline
\end{tabular}


medium were good as long as these polygons were not small. Of these polygons, only in $10 \%$ of cases was the agreement deemed to be poor.

We must assess the validation results with caution, for several reasons. One reason is that the dataset does not cover the whole winter with regular intervals. Another reason is the limited number of suitable AARI ice charts. Five of the seven nearly coincident datasets were acquired in March. This probably led to a slight bias in the results. In the available sample set, $67 \%$ of polygons were correctly identified (Table 2). On the basis of the KS statistics, in $49 \%$ of cases the frequency distributions were consistent (Fig. 8). The results suggest that the accuracy of our ice thickness estimation method is good for the FYI thin and FYI medium ice categories.

A larger and temporally more evenly spread set of AARI ice charts also covering early winter would improve the accuracy assessment. We expect difficulties in the detection of thin-ice areas in the $h_{\mathrm{ch}}$ chart. The most effective way to detect these areas is to use the MODIS $h_{\mathrm{i}}^{\mathrm{M}}$. If a thin-ice area is covered by clouds but is large enough, it can sometimes be identified relying on AMSR-E data ( $W$ matrix) or SAR data. However, our method often fails to detect thin-ice areas. On the basis of our limited validation data, we conclude that the proposed method gives realistic results in the thickness range $30-120 \mathrm{~cm}$ and is uncertain for very thin ice areas $(<30 \mathrm{~cm})$.

During our validation process the inaccuracies in the AARI ice charts were not taken into account. As mentioned earlier, the level of accuracy is not quantitatively known. This is an additional caveat for the validation results. Validation data including field measurements are required for a truthful accuracy assessment. The best validation data would be a representative set of ice thickness measurements based on electromagnetic induction as in Haas and others (1999).

\section{CONCLUSIONS}

Currently no single satellite instrument is able to provide us with a near-real-time view of the spatial distribution of the ice mass with accurate ice thickness estimates, for both thin level ice and thick deformed ice. We have presented a method using multisensor satellite data and a HIGHTSI seaice thermodynamic model for estimation of the spatial distribution of the thin-ice fields $(<1.2 \mathrm{~m}$ in this dataset) present in the Arctic FYI zone.

We have utilized the strengths of each satellite sensor. The ice thickness up to $40 \mathrm{~cm}$ is retrieved using MODIS ice surface temperature data and HIRLAM forcing data for cloudfree area. In cloud-covered regions, thin ice is located using the matrix $W$ derived from daily ice concentration changes, and a coarse ice thickness estimate is assigned. For thicker ice, thickness is retrieved from a combination of Envisat SAR image and modeled ice thickness background $H_{\mathrm{b}}$, which consists of thermodynamically modeled $H_{\mathrm{i}}$ field modified by the matrix $W$. The accuracy of thin-ice detection is at its best if the thin-ice area is cloud-free. According to the validation exercise, the joint SAR $\sigma^{0}$ mapping together with $H_{\mathrm{b}}$ produced realistic results for the spatial distribution of $\mathrm{FYI}$ thin-ice $(30-70 \mathrm{~cm})$ and medium ice $(70-120 \mathrm{~cm})$ types.

The obtained $h_{\mathrm{ch}}$ charts were validated against the frequency distributions of three sea-ice types which were extracted from seven weekly AARI ice charts. Altogether we had 84 ice chart polygons describing ice conditions.
According to the validation results for the $h_{\mathrm{ch}}$ charts, the FYI thin-ice and medium ice types were well identified. In this relatively small validation dataset, the accuracy in the ice thickness category classification varied around $80 \%$ depending on the applied accuracy criterion if the size of the polygon exceeded $20 \mathrm{~km}^{2}$. The detection of thin-ice polygons $(<30 \mathrm{~cm})$ succeeded only rarely, mainly because these polygons were typically very small. We also noted that about every fifth $h_{\text {ch }}$ chart contained anomalies due to the MODIS-based $h_{\mathrm{i}}$ regions. We did not address the uncertainties present in our reference dataset. Hence our accuracy assessments are indicative in nature. For the total accuracy assessment, further validation work is needed.

Our method is not applicable for operative use due to the infrequency of the cloud-free MODIS data. In order to provide an operative method we have carried out experiments to locate thin-ice areas (thickness less than 30-40 cm) based on the AMSR-E data (von Lerber and others, 2012). We continue to test different approaches to utilize the AMSR-E data in thin-ice identification so that a $h_{\mathrm{ch}}$ chart could be computed daily. A campaign will be carried out where $h_{\text {ch }}$ charts will be produced regularly during winter 2013/14 using AMSR data, and field observations will be collected. The current approach is, however, suitable to record the ice season evolution in the context of climate studies. We are studying how to incorporate ice drift (Karvonen, 2012) into our ice thickness estimation. One alternative is to utilize the modeled ice thickness fields of the mesoscale TOPAZ ocean/sea-ice model (Bertino and Lisæter, 2008), which already contain sea-ice dynamics.

\section{ACKNOWLEDGEMENTS}

This work was performed under the project Sea ice and snow products for the Barents, Pechora and Kara Seas using multisensor satellite data (KaraX) funded partly by the Finnish Funding Agency for Technology and Innovation (TEKES). We thank Juha Karvonen for computing the ice motion fields. We also thank the two anonymous reviewers and Sebastian Gerland for insightful comments and their efforts to improve the paper.

\section{REFERENCES}

Bertino L and Lisæter KA (2008) The TOPAZ monitoring and prediction system for the Atlantic and Arctic Oceans. J. Oper. Ocean., 1(2), 15-18

Bushuev AV and Loshchilov VS (2007) Ice chart composition at AARI. In Johannessen OM and 9 others eds. Remote sensing of sea ice in the northern sea route: studies and applications. (Nansen Center Polar Series 4) Springer-Praxis, Berlin, 243-252

Carlström A and Ulander LMH (1995) Validation of backscatter models for level and deformed sea-ice in ERS-1 SAR images. Int. J. Remote Sens., 16(17), 3245-3266 (doi: 10.1080/ 01431169508954629)

Cheng B, Launiainen J and Vihma T (2003) Modelling of superimposed ice formation and subsurface melting in the Baltic Sea. Geophysica, 39(1-2), 31-50

Cheng B and 6 others (2008) Model experiments on snow and ice thermodynamics in the Arctic Ocean with CHINARE 2003 data. J. Geophys. Res., 113(C9), C09020 (doi: 10.1029/ 2007JC004654)

Cheng B, Mäkynen M, Similä M, Rontu L and Vihma T (2013) Modelling snow and ice thickness in a coastal region of the Kara Sea, Arctic Russia. Ann. Glaciol., 54(62 Pt 1), 105-113 (doi: 10.3189/2013AoG62A180) 
Dierking W, Pettersson MI and Askne J (1999) Multifrequency scatterometer measurements of Baltic Sea ice during EMAC-95. Int. J. Remote Sens., 20(2), 349-372 (doi: 10.1080/ 014311699213488)

Divine DV, Korsnes R, Makshtas AP, Godtliebsen F and Svendsen H (2005) Atmospheric-driven state transfer of shore-fast ice in the northeastern Kara Sea. J. Geophys. Res., 110(C9), C09013 (doi: 10.1029/2004JC002706)

Drucker R, Martin S and Moritz R (2003) Observations of ice thickness and frazil ice in the St. Lawrence Island polynya from satellite imagery, upward looking sonar, and salinity/temperature moorings. J. Geophys. Res., 108(C5), 3149 (doi: 10.1029/ 2001JC001213)

Dudewicz EJ and Mishra SN (1988) Modern mathematical statistics. Wiley, Singapore

European Space Agency (ESA) (2009) ENVISAT ASAR monthly report. (Tech. Note ENVI-CLVL-EOPG-TN-04-0009) European Space Agency, Noordwijk

Frey RA and 6 others (2008) Cloud detection with MODIS. Part I: Improvements in the MODIS cloud mask for Collection 5. J. Atmos. Ocean. Technol., 25(7), 1057-1072 (doi: 10.1175/ 2008JTECHA1052.1)

Haas C, Rupp KH and Uuskallio A (1999) Comparison of along track EM ice thickness profiles with ship performance data. In Tuhkuri J and Riska K eds. Proceedings of the 15th International Conference on Port and Ocean Engineering under Arctic Conditions (POAC'99), 23-27 August 1999, Espoo, Finland. Geophysical Institute, University of Alaska Fairbanks, Fairbanks, AK, 343-353

Johannessen OM and 9 others. (2007) Remote sensing of sea ice in the northern sea route: studies and applications. (Nansen Center Polar Series 4) Springer-Praxis, Berlin

Kaleschke L, Tian-Kunze X, Maaß N, Mäkynen M and Drusch M (2012) Sea ice thickness retrieval from SMOS brightness temperatures during the Arctic freeze-up period. Geophys. Res. Lett., 39(5), L05501 (doi: 10.1029/2012GL050916)

Källén E ed (1996) HIRLAM documentation manual, system 2.5. Swedish Meteorological and Hydrological Institute, Norrköping

Karvonen J (2012) Operational SAR-based sea ice drift monitoring over the Baltic Sea. Ocean Sci., 9(1), 359-384 (doi: 10.5194/ osd-9-359-2012)

Karvonen JA, Similä MH and Istvan H (2003) Ice thickness estimation using SAR data and ice thickness history. In International Geoscience and Remote Sensing Symposium (IGARSS 2003), 21-25 July 2003, Toulouse, France. Vol. 1. Institute of Electrical and Electronics Engineers, Piscataway, NJ, 74-76

Karvonen JA, Similä MH, Haapala J, Haas C and Mäkynen MP (2004) Comparison of SAR data and operational sea ice products to EM ice thickness measurements in the Baltic Sea. In International Geoscience and Remote Sensing Symposium (IGARSS 2004), 2024 September 2004, Anchorage, AK, USA. Vol. 5. Institute of Electrical and Electronics Engineers, Piscataway, NJ, 3012-3024

Karvonen JA, Haapala J, Lehtiranta J and Seinä A (2007) Polarview@FIMR: WWW-based delivery of Baltic sea ice products to end-users. In International Geoscience and Remote Sensing Symposium (IGARSS 2007), 23-28 July 2007, BarceIona, Spain. Institute of Electrical and Electronics Engineers, Piscataway, NJ, 1242-1245

Kwok R and Cunningham GF (1994) Backscatter characteristics of the winter ice cover in the Beaufort Sea. J. Geophys. Res., 99(C4), 7787-7802 (doi: 10.1029/94JC00169)

Kwok R and Rothrock DA (2009) Decline in Arctic sea ice thickness from submarine and ICESat records: 1958-2008. Geophys. Res. Lett., 36(15), L15501 (doi: 10.1029/2009GL039035)

Kwok R, Nghiem SV, Yueh SH and Huynh DD (1995) Retrieval of thin ice thickness from multifrequency polarimetric SAR data. Remote Sens. Environ., 51(3), 361-374 (doi: 10.1016/00344257(94)00017-H)

Launiainen J and Cheng B (1998) Modelling of ice thermodynamics in natural water bodies. Cold Reg. Sci. Technol., 27(3), 153-178 (doi: 10.1016/S0165-232X(98)00009-3)
Laxon S, Peacock N and Smith D (2003) High interannual variability in sea ice thickness in the Arctic region. Nature, 425(6961), 947-950 (doi: 10.1038/nature02050)

Leppäranta M (2005) The drift of sea ice. Springer, Berlin

Lundhaug M (2002) ERS SAR studies of sea ice signatures in the Pechora Sea and Kara Sea region. Can. J. Remote Sens., 28(2), 114-127 (doi: 10.5589/m02-022)

Maaß N and Kaleschke L (2010) Improving passive microwave sea ice concentration algorithms for coastal areas: applications to the Baltic Sea. Tellus A, 62(4), 393-410 (doi: 10.1111/j.16000870.2010.00452.x)

Mäkynen MP, Manninen AT, Similä MH, Karvonen JA and Hallikainen MT (2002) Incidence angle dependence of the statistical properties of C-band $\mathrm{HH}$-polarization backscattering signatures of the Baltic Sea ice. IEEE Trans. Geosci. Remote Sens., 40(12), 2593-2605 (doi: 10.1109/TGRS.2002.806991)

Mäkynen M, Similä M, Cheng B and Laine V (2010a) On level ice thickness retrieval in the Kara Sea using MODIS and ENVISAT ASAR data. In Lacoste-Francis $\mathrm{H}$ ed. Proceedings of the ESA Living Planet Symposium, 28 June-2 July 2010, Bergen, Norway. (ESA SP-686) European Space Agency, Noordwijk

Mäkynen M, Similä M, Cheng B, Laine V and Karvonen J (2010b) Sea ice thickness retrieval in the Baltic Sea using MODIS and SAR data. In Lacoste-Francis $\mathrm{H}$ ed. Proceedings of 3rd International Workshop on Advances in SAR Oceanography from Envisat and ERS Missions (SeaSAR 2010), 25-29 January 2010, Frascati, Italy. (ESA SP-679) European Space Agency, Noordwijk. CD-ROM

Mäkynen M, Cheng B and Similä M (2013) On the accuracy of the thin-ice thickness retrieval using MODIS thermal imagery over the Arctic first-year ice. Ann. Glaciol., 54(62 Pt 1), 87-96 (doi: 10.3189/2013AoG62A166)

Manninen T (1996) Microwave surface backscattering and surface roughness of Baltic Sea ice. Finn. Mar. Res. 265

Martin S, Drucker R, Kwok R and Holt B (2004) Estimation of the thin ice thickness and heat flux for the Chukchi Sea Alaskan coast polynya from Special Sensor Microwave/Imager data, 1990-2001. J. Geophys. Res., 109(C10), C10012 (doi: 10.1029/ 2004JC002428)

Maykut, GA (1986). The surface heat and mass balance. In Untersteiner N ed. Geophysics of sea ice (NATO ASI Series B: Physics 146) Plenum Press, London, 395-463

Nakamura K, Wakabayashi H, Uto S, Naoki K, Nishio F and Uratsuka S (2006) Sea-ice thickness retrieval in the Sea of Okhotsk using dual-polarization SAR data. Ann. Glaciol., 44, 261-268 (doi: 10.3189/172756406781811420)

Nakamura K, Wakabayashi H, Uto S, Ushio S and Nishio F (2009) Observation of sea-ice thickness using ENVISAT data from Lützow-Holm Bay, East Antarctica. IEEE Trans. Geosci. Remote Sens., 6(2), 277-281 (doi: 10.1109/LGRS.2008.2011061)

Nihashi S, Ohshima KI, Tamura T, Fukamachi Y and Saitoh S (2009) Thickness and production of sea ice in the Okhotsk Sea coastal polynyas from AMSR-E. J. Geophys. Res., 114(C10), C10025 (doi: 10.1029/2008JC005222)

Onstott RG (1992) SAR and scatterometer signatures of sea ice. In Carsey FD and 7 others eds. Microwave remote sensing of sea ice (Geophysical Monograph Series 68) American Geophysical Union, Washington, DC, 73-104

Similä M, Mäkynen M and Heiler I (2010) Comparison between C band synthetic aperture radar and 3-D laser scanner statistics for the Baltic Sea ice. J. Geophys. Res., 115(C10), C10056 (doi: 10.1029/2009JC005970)

Spreen G, Kaleschke L and Heygster G (2008) Sea ice remote sensing using AMSR-E 89-GHz channels. J. Geophys. Res., 113(C2), C02S03 (doi: 10.1029/2005JC003384)

Tamura T and Ohshima KI (2011) Mapping of sea ice production in the Arctic coastal polynyas. J. Geophys. Res., 116(C7), C07030 (doi: 10.1029/2010JC006586)

Tamura T and 6 others (2006) Estimation of thin sea-ice thickness from NOAA AVHRR data in a polynya off the Wilkes Land coast, 
East Antarctica. Ann. Glaciol., 44, 269-274 (doi: 10.3189/ 172756406781811745)

Undén P and 26 others. (2002) The high resolution limited area model. HIRLAM-5 scientific documentation. Swedish Meteorological and Hydrological Institute, Norrköping

Von Lerber A, Mäkynen M, Similä M, Sievinen P and Hallikainen MT (2012) Thin sea ice identification in the Kara Sea using AMSR-E data. In International Geoscience and Remote Sensing Symposium (IGARSS 2012), 22-27 July 2012, Munich, Germany. Institute of Electrical and Electronics Engineers, Piscataway, NJ, 4477-4480

Wakabayashi H, Matsuoka T, Nakamura K and Nishio F (2004) Polarimetric characteristics of sea ice in the Sea of Okhotsk observed by airborne L-band SAR. IEEE Trans. Geosci. Remote Sens., 42(11), 2412-2425 (doi: 10.1029/JZ069i022p04755)

World Meteorological Organization (WMO) (1989) WMO sea-ice nomenclature: terminology, codes and illustrated glossary. (WMO/OMM/BMO Report 259) World Meteorological Organization, Geneva

Yu Y and Rothrock DA (1996) Thin ice thickness from satellite thermal imagery. J. Geophys. Res., 101(C11), 25 753-25 766 (doi: 10.1029/96JC02242)

Zakhvatkina NY, Alexandrov VY, Johannessen OM, Sandven S and Frolov IY (in press) Classification of sea ice types in ENVISAT synthetic aperture radar images. IEEE Trans. Geosci. Remote Sens. (doi: 10.1109/TGRS.2012.2212445)

\section{APPENDIX}

Here we describe the determination of the elements $w$ in the $W$ matrix ('ice concentration (IC) filter coefficients') introduced in Section 4.3. The $w$ coefficients are determined for the following ice concentration ranges: IC $\leq 35 \%$ (denoted as IC35), [35\% < IC <80\%] (IC50), [80\% $\leq$ IC $<90 \%$ ] (IC85), $[90 \% \leq$ IC $<95 \%$ ] (IC92), with the following geophysically motivated rules:

1. If IC belonged to IC92 or IC80 during the 5 days prior to the analysis day, we set $w=0.95$ and $w=0.9$, respectively. This situation is possible everywhere in the icecovered area.

2. If IC belonged to IC50, the value of $w$ depends on when this event last occurred and how long it lasted. If it occurred just once during the previous 5 days we set $w=0.5$. If it occurred more often, we set $w=0.45$. This situation is typical for polynyas or the ice edge area.

3. IC values below $35 \%$ are considered to represent open water. The oldest occurrence of open water taken into account is that during the previous $11-14$ days $(w=0.5)$. The thickness of thermodynamically grown ice then remains $<40 \mathrm{~cm}$ even in cold polar conditions after 10 12 days (Maykut, 1986). If the last occurrence of open water took place 5-10 days prior to the analysis, we set $w=0.4$. If it was 3-5 or 1-2 days prior to the classification, we set $h_{\mathrm{i}}$ to 20 and $10 \mathrm{~cm}$, respectively. We use these approximative $h_{\mathrm{i}}$ values because they are sufficient for thin-ice area identification, which is our main target.

4. For the IC chart on the analysis day, we set $w$ to $0.9,0.8$, $0.6,0.35$ or 0 if the corresponding IC belongs to ranges IC92, IC $85, \quad[60 \% \leq \mathrm{IC}<80 \%], \quad[35 \%<\mathrm{IC}<60 \%]$ or IC35, respectively. On the analysis day we divide the IC50 range into two parts because the current IC chart contains fewer uncertainties concerning the prevailing ice situation than the older IC charts.

5. Two matrices $W_{\text {history }}$ and $W_{\text {today }}$ are constructed. The former is based on the IC history during the last 14 days, and the latter on the IC situation on the analysis day. The matrix $W$ used in the analysis is $W=\min \left(W_{\text {history, }}\right.$ $\left.W_{\text {today }}\right)$.

On the analysis day the $w$ value for a given ice concentration value is slightly smaller than in the historical data. If ice concentration in the area of interest remains at the same, or lower, level in the current ice concentration chart as in the historical ice concentration data, then the newest $w$ value is selected. Otherwise, ice concentration has increased in the area and the somewhat larger $w$ value is justified.

After smoothing the $w$ values by taking the mean value over a sliding window $(3 \times 3$ pixels), we obtain a modified background ice thickness field $H_{\mathrm{b}}$ with Eqn (1). Although $W$ before the averaging has the same resolution as the ice concentration chart $(6.25 \mathrm{~km})$, the actual resolution of $H_{\mathrm{b}}$ is much larger because of the smoothing and $20 \mathrm{~km}$ resolution of $H_{\mathrm{i}}$. Prior to the analysis, $H_{\mathrm{b}}$ is interpolated to the same grid as the SAR data $(2 \mathrm{~km})$. 